\title{
Programa de capacitação de servidores e sustentabilidade: uma análise na Universidade Federal do Tocantins, Brasil
}

\section{Gilberto Soares da Silva}

Universidade Federal do Tocantins. Campus de Araguaína. Av. Paraguai, S/№. Bairro da Cimba. Araguaína-TO, Brasil (CEP 77824838). E-mail: gilbertosoares@mail.uft.edu.br.

Resumo. 0 cenário atual no âmbito das organizações e também no setor público aponta para a necessidade de inovação como diferencial, num contexto de mudanças constantes. Nessa conjuntura, ressalta-se que o quadro de pessoal possui papel primordial, uma vez que realiza o papel estratégico nas organizações. Outra temática emergente, e que vem crescendo a cada dia nos debates internacionais é a questão da sustentabilidade. Neste sentido, buscou-se analisar se a Universidade Federal do Tocantins (UFT) tem se sensibilizado na oferta de capacitação aos seus servidores com temáticas voltadas para a sustentabilidade. Metodologicamente, foi realizada uma revisão de literatura, e análise documental no âmbito da UFT, de modo a verificar o planejamento dos cursos de capacitação e a oferta dos mesmos, analisando a sensibilização institucional na oferta de cursos com abordagem socioambiental. A pesquisa se caracteriza como um estudo de caso, com abordagem qualitativa. Como principais resultados, destaca-se, que a UFT possui um programa de capacitação bem elaborado em todos os campi, com previsão e oferta de cursos aos servidores, e baseado nas lacunas informadas pelos próprios servidores. Com relação aos cursos voltados às temáticas socioambientais, observa-se que são previstos no planejamento e ofertados alguns cursos de capacitação relacionados ao tema, destaca-se, ainda, que a Instituição pode elaborar um programa de cursos específicos sobre sustentabilidade para a formação de seu quadro de pessoal, dada a importância da temática.

Palavras-chave: Ações de capacitação; Sustentabilidade; Universidades.

Abstract. Server and sustainability training program: An analysis at the Federal University of Tocantins, Brazil. The current scenario in organizations and also in the public sector points to the need for innovation as a differential, in a context of constant changes. At this juncture, it is emphasized that the staff has a primordial role, since it plays the strategic role in organizations. Another emerging issue that has been growing every day in international debates is the question of
Recebido:

24/10/2018

Aceito:

23/12/2018

Publicado:

$31 / 12 / 2018$

Acesso aberto

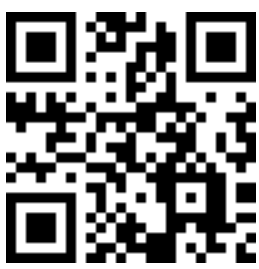

ORCID

(1) 0000-0003-4595-9031 Gilberto Soares da Silva 
sustainability. In this sense, the aim was to analyze whether the Federal University of Tocantins (UFT) has been sensitized in offering training to its servers with themes focused on sustainability. Methodologically, a review of literature and documentary analysis was carried out within the scope of the UFT, in order to verify the planning of the training courses and their offer, analyzing the institutional awareness in the offer of courses with a socio-environmental approach. The research is characterized as a case study with a qualitative approach. As main results, it is worth noting that UFT has a well-developed training program in all campi, with provision and provision of courses to the servers, and based on the gaps reported by the servers themselves. With regard to the courses on social and environmental themes, it is noted that some training courses related to the theme are planned and offered, and it is also worth noting that the Institution can elaborate a program of specific courses on sustainability for the formation of its staff, given the importance of the theme.

Keywords: Capacity building actions; Sustainability; Universities.

\section{Introdução}

A sustentabilidade vem crescendo nos debates internacionais e se insere na realidade das organizações e instituições (Cole, 2003). Tendo em vista a necessidade de atendimento as demandas de capacitação de servidores com fulcro nos regulamentos conforme a Lei $\mathrm{n}^{-}$11.091/2005 (Brasil, 2005), que trata dos Planos de Carreira dos Cargos Técnico-Administrativos em Educação (PDIPCCTAE), e a Política Nacional de Desenvolvimento de Pessoal (PNDP), procurou-se neste artigo, discutir a realidade de uma instituição de Ensino Superior e a oferta de capacitações voltadas para a sustentabilidade.

Este artigo buscou analisar se a Universidade Federal do Tocantins (UFT) tem se sensibilizado na oferta de capacitação aos seus servidores com temáticas voltadas para a sustentabilidade. A ideia central é averiguar as políticas de capacitação de servidores no âmbito da UFT, e constatar se de algum modo a Instituição promove ações de capacitação voltadas para temáticas sustentáveis.

0 presente trabalho se justifica na necessidade de as Instituições realizarem ações de capacitação atreladas a uma política de Gestão por Competências, conforme as diretrizes e regulamentos governamentais. Destarte, verifica-se, ainda, a abordagem socioambiental presente em tais ações, tendo em vista o crescente debate sobre sustentabilidade entre diferentes camadas sociais.

Assim, este artigo encontra-se estruturado em quatro partes principais, excluída esta introdução e as referências bibliográficas. A primeira aborda a fundamentação teórica para o assunto aqui discutido. Em seguida, na segunda parte, fundamenta-se a metodologia e o percurso percorrido. Na quarta parte, trata-se da apresentação e discussão dos resultados encontrados, e por fim, a última parte traz algumas considerações finais.

Política Nacional De
Desenvolvimento de Pessoas
Em cumprimento ao que está
previsto no PDIPCCTAE, conforme a Lei
no 11.091/2005 (Brasil, 2005), bem
como, nos Planos de Formação e
Desenvolvimento (PDF) da Universidade
Federal do Tocantins, são realizadas
ações de capacitação para os servidores,
com a finalidade de desenvolver


capacidades e habilidades essenciais para o desempenho profissional $\mathrm{e}$ pessoal do quadro de servidores, resultando em prestação de serviços de qualidade à comunidade.

Neste sentido, cita-se a importância de ter uma matriz de capacitação atrelada à PNDP da Administração Pública Federal. Faz-se necessário ter uma política voltada para a gestão por competências, em atendimento às prioridades governamentais e o desenvolvimento de competências em toda a administração pública.

Nesta perspectiva, Dutra (2004) informa que a frequente utilização do termo competência no campo organizacional, tendo sido empregado de diversas e variadas maneiras; destarte, pode-se observar no campo teórico que tais conceitos se amparam por distintas concepções epistemológicas. Por um lado, observa-se uma corrente de teóricos, a exemplo de McClelland (1973) e Boyatzis (1982), que entendem as competências voltadas para as características e traços individuais, ou ainda, para as atribuições que as pessoas desenvolvem de acordo com seus desempenhos. E, a outra vertente, é defendida por Fleury e Fleury (2001), Zarifian (2001) e Le Boterf (2003), que se fundamentam num aspecto mais integrativo e interpretativista, levando em conta uma dimensão socialmente construída personificada num ambiente onde as competências são expressas, sendo este o conceito empregado neste artigo.

No caso dos programas de capacitação, no âmbito do setor público, estes devem estar atrelados diretamente a um programa de Gestão por competências. No caso da UFT, preconiza-se que o Plano de Formação dos servidores tem por cerne colaborar para a eficiência do serviço público, bem como dos seus servidores. Para isso, devem ser realizadas ações de capacitação e de desenvolvimento pessoal ordenado e de modo contínuo, que legitimem os interesses da
Instituição e o desenvolvimento pessoal/ profissional, aprimorando, deste modo, os recursos humanos e orçamentários disponíveis (UFT, 2017).

De acordo com Schikmann (2010), desenvolver a gestão de pessoas de modo estratégico, requer 0 atendimento de alguns mecanismos e instrumentos, tais como "a) Planejamento de recursos humanos; b) Gestão de competências; c) Capacitação continuada com base em competências; e d) Avaliação de desempenho e de competências". Com essas ferramentas, a autora ressalta, ainda, que a capacitação tem por intuito desenvolver o quadro de pessoal, de modo que se desenvolvam as competências necessárias para atingir às necessidades e metas institucionais. Com isso, visa-se ao melhor desempenho institucional e a efetivação dos planos e metas definidos pelo planejamento da Instituição.

As ações de capacitação previstas no Plano da UFT preveem o atendimento às lacunas mapeadas de acordo com as necessidades setoriais. De acordo com Silva e Silva (2018), um programa de capacitação eficaz e eficiente, necessita do apoio de uma gestão sensível às verdadeiras necessidades setoriais, e o seu planejamento deve ser integrado, participativo, de modo dinâmico, por meio de mecanismos que permitam uma avaliação contínua e aberta a todos os interessados.

Portanto, percebe-se a importância que as ações de capacitação possuem para o desenvolvimento pessoal/ profissional e institucional. Salienta-se, ainda, que as instituições devem planejar, coordenar e avaliar as competências de seu quadro de pessoal, por meio do desenvolvimento permanente dos conhecimentos e habilidades necessárias para o desempenho profissional.

A sustentabilidade nas instituições de ensino superior

Tendo em vista a importância dos programas de capacitação institucional, e em face das alterações que o mundo vem 
sofrendo com relação à escassez de recursos naturais e outras demandas, faz-se necessário repensar algumas práticas no campo da produção, abordando frentes não apenas econômicas.

Carece aqui, definir o que se entende por sustentabilidade, e, utilizando-se do conceito da Comissão Mundial sobre Meio Ambiente e Desenvolvimento (CMMAD) acerca do desenvolvimento sustentável, ressalta-se que a sustentabilidade abriga paradigmas para a utilização dos recursos que intenta atender às necessidades dos seres humanos. 0 termo desenvolvimento sustentável foi utilizado no Relatório Brundtland, que constituiu a base para sua construção, sendo este, o desenvolvimento que "satisfaz as necessidades do presente sem comprometer a capacidade das gerações futuras de satisfazerem as suas próprias necessidades" (CMMAD, 1988). Para Sachs (2008), a sustentabilidade possui oito vertentes essenciais que deverão ser alcançadas, quais sejam social, cultural, ecológica, ambiental, territorial, econômica, política Nacional e política Internacional.

Em virtude disso, as Instituições de Ensino Superior (IES) possuem um papel crucial na disseminação de serviços de qualidade na formação de perfis profissionais. Para Quadros (1999) há necessidade de se pensar em diálogos mais abertos e que promovam a qualidade de vida para a sociedade por meio do ensino, pesquisa e extensão. Voltando para a arena da sustentabilidade, Souza et al. (2011) apontam que as IES devem demonstrar sua contribuição para refletir sobre mecanismos de produção e gerencial, de modo que suas ações sejam mais sustentáveis.

Em Sachs (2009), observa-se que a sustentabilidade surgiu como uma possível solução entre a dicotômica vertente puramente econômica e o fundamentalismo ambiental. Esse conceito de sustentabilidade perpassa por três pilares essenciais, que são: a importância social, a prevenção ecológica e a possibilidade econômica. $\mathrm{O}$ autor compreende que o crescimento econômico é necessário e fundamental, entretanto, ele precisa permitir cuidados com a dimensão social, e deve ser atingido através de práticas que não destruam o meio ambiente natural. Cole (2003) assegura que as Instituições de Ensino sustentáveis são aquelas que consentem que a comunidade institucional tenha costumes que resguardem e melhorem a saúde e a qualidade de vida da comunidade interna e externa, como também de seu meio natural.

Mediante o exposto, a sustentabilidade deve ser alcançada por todas as organizações e instituições, sejam elas sociais ou não. Neste sentido, o Estado também possui papel preponderante no processo de conscientização da sociedade e estímulo para que os demais agentes busquem o desenvolvimento sustentável. Desse modo, o aparelho estatal atua como indutor de políticas que apontem para o desenvolvimento sustentável, atuando diretamente na elaboração, implementação e divulgação de medidas que efetivem a sustentabilidade.

\section{Agenda ambiental da administração pública e planos de gestão e logística sustentável}

No caso brasileiro, criou-se a

Agenda Ambiental da Administração Pública (A3P), elaborada com base nos princípios da Agenda 21, princípio oito da Declaração do Rio'92 e na Declaração de Johanesburgo. Estas declarações asseguram que os países necessitam formar e realizar a avaliação e realizar novos padrões de sustentabilidade, por meio de políticas públicas e táticas que admitam novos resultados de produção e consumo, fundamentais para 0 desenvolvimento sustentável (Cole, 2003).

Assim, para atingir os objetivos da Agenda, são recomendadas na A3P 
ações que dão prioridade à política dos 5R's, que são: Repensar, Reduzir, Reaproveitar, Reciclar e Recusar. Para que essa política se torne efetiva, encontra-se estruturada em cinco eixos temáticos principais, o uso racional dos recursos naturais e bens públicos, a gestão adequada dos resíduos gerados, a qualidade de vida no ambiente de trabalho, a sensibilização e capacitação dos servidores, e as licitações sustentáveis (Brasil, 2009).

Como o foco da pesquisa fundamenta-se no item quatro, entendese que o mesmo necessita de maiores detalhes. De acordo com a A3P, a sensibilização e capacitação dos servidores dos órgãos públicos, busca implicar na mudança dos hábitos do quadro de pessoal, da conduta e dos modos de consumo. Para isto, procuramse propor algumas ações necessárias para atingir os resultados, quais sejam, criar e consolidar nos servidores a consciência cidadã da responsabilidade socioambiental por meio de campanhas, palestras, minicursos, apresentações, fóruns, e a capacitação, principalmente dos gestores (Brasil, 2009).

Como modo de regulamentar essa política nos órgãos públicos, surgiu a Instrução Normativa no 10/2012 (Brasil, 2012), que instituiu normas para a elaboração do Plano de Gestão e Logística Sustentável (PLS), pelos órgãos da Administração Pública Federal, observando práticas de sustentabilidade e o uso racional de produtos e processos nas entidades públicas.

A primeira seção da IN $n^{\underline{0}}$ 10/2012 estabelece os critérios gerais de elaboração, e, no que se refere aos conteúdos a serem abordados, o art. 5o especifica, que devem abranger também, a divulgação, conscientização e capacitação dos recursos humanos (Brasil, 2012). De acordo com as práticas de sustentabilidade e racionalização do uso de materiais e serviços, previstos na IN no 10/2012, o art. 8o estabelece que devam conter, no mínimo, as seguintes abordagens: racionalização dos materiais de expediente; fontes de energia, água e esgoto; tratamento adequado para resíduos; ações de qualidade de vida, saúde e segurança no ambiente de trabalho; licitação de compras sustentáveis; e, redução de emissão de gases poluentes (Brasil, 2012).

A sustentabilidade permeia a mudança de hábitos no ambiente organizacional, portanto, considerando a proposta deste estudo, buscar-se-á analisar os cursos de capacitação planejados e ofertados na UFT. Os conteúdos e abordagens previstos na A3P e IN no 10/2012 servirão como norteadores para a análise e discussão dos resultados da pesquisa.

\section{Metodologia}

Esta pesquisa se caracteriza como um estudo de caso, pois, buscou analisar se a Universidade Federal do Tocantins tem se sensibilizado na oferta de capacitação aos seus servidores com temáticas voltadas para a sustentabilidade. Para Prodanov e Freitas (2013)

estudo de caso consiste em coletar e analisar informações sobre determinado indivíduo, uma família, um grupo ou uma comunidade, a fim de estudar aspectos variados de sua vida, de acordo com o assunto da pesquisa.

No caso desta pesquisa, o estudo de caso busca analisar o programa de capacitação de servidores da Universidade Federal do Tocantins, no ano de 2017. O trabalho se pauta na importância tanto das competências a serem alcançadas pelo programa de capacitação, como também, pela urgência de se pensar e sensibilizar enquanto agentes responsáveis pela sustentabilidade.

Para atingir os objetivos deste estudo, o percurso metodológico foi baseado em duas partes principais: a revisão de literatura e a análise 
documental. A primeira buscou em fontes de outros pesquisadores, fundamentar a teoria a ser aplicada na proposta desta pesquisa, que de acordo com Gil (2008) é um processo fundamental em todo tipo de pesquisa. A segunda levantou dados junto aos Planos de Formação e Desenvolvimento (PFD) da UFT, em busca dos dados necessários que a pesquisa propõe. Para Beuren (2006), a pesquisa documental é interessante por poder reunir informações, até então, estruturadas ou não, tornando-as importantes dados e fontes para outras pesquisas. As informações foram levantadas junto aos PFD da UFT e dados contidos no Sistema de Informações para o Ensino (SIE), com a relação de cursos ofertados em 2017 (UFT, 2018).

Para a fase da análise, propôs-se uma abordagem qualitativa. A escolha por essa abordagem se deu conforme o que propõe Prodanov e Freitas (2013), onde estudos qualitativos são de fundamental importância para refletir realidades sobre determinada temática. A proposta visou a indicar o quantitativo de cursos ofertados, analisando-os qualitativamente, no intuito de anteder aos objetivos da pesquisa. Para essa fase da pesquisa, utilizaram-se os requisitos propostos pela literatura sobre as dimensões da sustentabilidade: social; ambiental; e, econômico; bem como, os princípios e diretrizes da A3P e IN no 10/2012.

\section{Resultados e discussão}

A pesquisa foi realizada tendo como objeto de estudo a Universidade Federal do Tocantins (UFT), criada em 23 de outubro de 2000, iniciando suas atividades em maio de 2003. Hoje a instituição conta com um quadro de 1.051 servidores docentes, 829 técnicoadministrativos. A Universidade está estruturada em um sistema multicampi, localizada em regiões estratégicas do estado tocantinense, nas cidades de Araguaína, Arraias, Gurupi, Miracema,
Palmas, Porto Nacional e Tocantinópolis. Ressalta-se que, a Reitoria fica localizada na capital do estado, Palmas (UFT, 2016).

Em análise aos documentos institucionais, salienta-se que a UFT busca contribuir, de modo expresso, com o desenvolvimento socioambiental no contexto regional no qual está inserida, ao propor como missão "formar profissionais cidadãos e produzir conhecimentos com inovação e qualidade que contribuam para o desenvolvimento socioambiental do Estado do Tocantins e da Amazônia Legal" (UFT, 2016).

Para esta fase da pesquisa os dados foram coletados, primeiramente, junto ao PFD dos sete campi e reitoria, mapeando o modelo e os cursos previstos para oferta no biênio 20172018. No entanto, consideraram-se, na segunda fase, os cursos ofertados no ano de 2017, coletados junto ao SIE. Para melhor descrever os dados foram distribuídos de acordo com cada campus e reitoria.

Para elaboração do PFD a UFT utiliza como base, além, das diretrizes nacionais, a Resolução do Conselho Universitário (CONSUNI) no 20/2015, que estabelece as normas para a elaboração do Programa de Capacitação de servidores no âmbito da Instituição. 0 programa de capacitação da UFT é ofertado nas seguintes modalidades: treinamento em serviço, grupo formal de estudo, estágio profissional, visitas técnicas e eventos de curta duração (congressos, encontros, conferências, seminários, fóruns, mesas-redondas, palestras, oficinas ou similares) (UFT, 2015).

Os planos de formação da UFT possuem ainda, algumas linhas de desenvolvimento, que visam atender: a iniciação ao serviço público, capacitação geral, práticas de gestão, interambientes e capacitações específicas de cada cargo/setor. Percebeu-se que para atendimento dos programas de capacitação, a UFT instituiu o Programa Servidor Multiplicador, cujo objetivo é a disseminação de conhecimento entre os 
próprios servidores. São realizados na modalidade de capacitação interna, onde os servidores do próprio quadro replicam suas habilidades para o quadro de servidores que possuem lacunas em determinadas competências.

0 que se observa de positivo é que a Instituição possui um Programa de Capacitação bem regulamentado, tendo como proposta sua elaboração a cada dois anos, e com base nas lacunas mapeadas pelos próprios servidores. A existência do PFD em cada campus, bem como, a Resolução do CONSUNI no 23/2015 regulamentando, demonstram que a UFT tem se preocupado com o planejamento de ações que visem diminuir as lacunas destacadas pelo mapeamento setorial.

Em relação ao total de cursos previstos no PFD, encontram-se na Tabela 1, os dados da UFT, distribuídos por cada campus.

Tabela 1. Distribuição dos cursos a serem ofertados por campus na UFT.

\begin{tabular}{|l|c|}
\hline Campus & Total de Cursos \\
\hline Araguaína & 50 \\
\hline Arraias & 15 \\
\hline Gurupi & 47 \\
\hline Miracema & 17 \\
\hline Palmas & 54 \\
\hline Porto Nacional & 37 \\
\hline Tocantinópolis & 15 \\
\hline Reitoria & 237 \\
\hline
\end{tabular}

Fonte: Adaptado de UFT (2017).

Observa-se que a programação de oferta é grande se forem considerados que os programas de capacitação são elaborados para cada dois anos. Assim, se cada campus ofertasse em média um curso por mês ainda não atenderia ao total de cursos previstos em alguns campi (Araguaína, Gurupi, Palmas, Porto Nacional e Reitoria). Ressalta-se, ainda, que os campi que propõem mais cursos são os que possuem o maior quadro de servidores, Reitoria, Palmas, Araguaína, Gurupi, Porto Nacional, Arraias, Tocantinópolis e Miracema, respectivamente (UFT, 2018).

Outra observação realizada na análise dos dados foi que dos 472 cursos previstos para oferta, mais de $65 \%$ são propostos na modalidade capacitação interna, sendo os demais distribuídos entre capacitação externa, treinamentos em serviço, grupo formal de estudos, cursos a distância e Programa de Formação Continuada docente (PROFOR).

Ao analisar se os cursos previstos possuem alguma relação com as temáticas da sustentabilidade, percebese que os dados apontam para um baixo número de cursos de capacitações previstos para oferta sobre questões socioambientais. Do total de cursos previstos, detectaram-se, apenas, sete cursos em Araguaína, dois em Gurupi, um em Miracema, cinco em Palmas, dois em Porto Nacional e seis na Reitoria.

Observou-se, ainda, que estes cursos previstos para oferta tratavam de temáticas voltadas para as três dimensões da sustentabilidade (social, ambiental e econômico), sobre as mais variadas temáticas. Foram identificados, sete cursos sobre questões relacionadas à segurança, saúde e qualidade de vida no ambiente de trabalho, bem como, questões de estímulo motivacional e bem-estar. No campo da dimensão ambiental, percebeu-se, 13 cursos previstos, dos quais se destacam abordagens sobre a conservação do patrimônio público, boa gestão dos materiais, educação, gestão e impactos ambientais, destinação de resíduos e mudanças de hábitos de consumo. Na esfera econômica, percebem-se, três propostas de cursos, sobre gestão orçamentária, financeira e patrimonial.

Deste modo, conforme estabelece a A3P 'acerca dos conteúdos a serem desempenhados pelos órgãos públicos, sobre questões sustentáveis, observa-se intenção de oferta de capacitação e 
sensibilização institucional voltada para temáticas socioambientais. No entanto, se comparados ao total de cursos previstos, ainda se pensa minimamente em questões sustentáveis, sem deixar de mencionar que dois dos sete campi não tiveram nenhum curso em áreas correlatas à sustentabilidade, na previsão de oferta.

Já com relação aos cursos ofertados na UFT no ano de 2017, observou-se, conforme a Figura 1, que no total foram atendidos 102 cursos, quase $25 \%$ dos cursos previstos para oferta na Instituição.

Figura1. Cursos de Capacitação ofertados na UFT, em 2017.

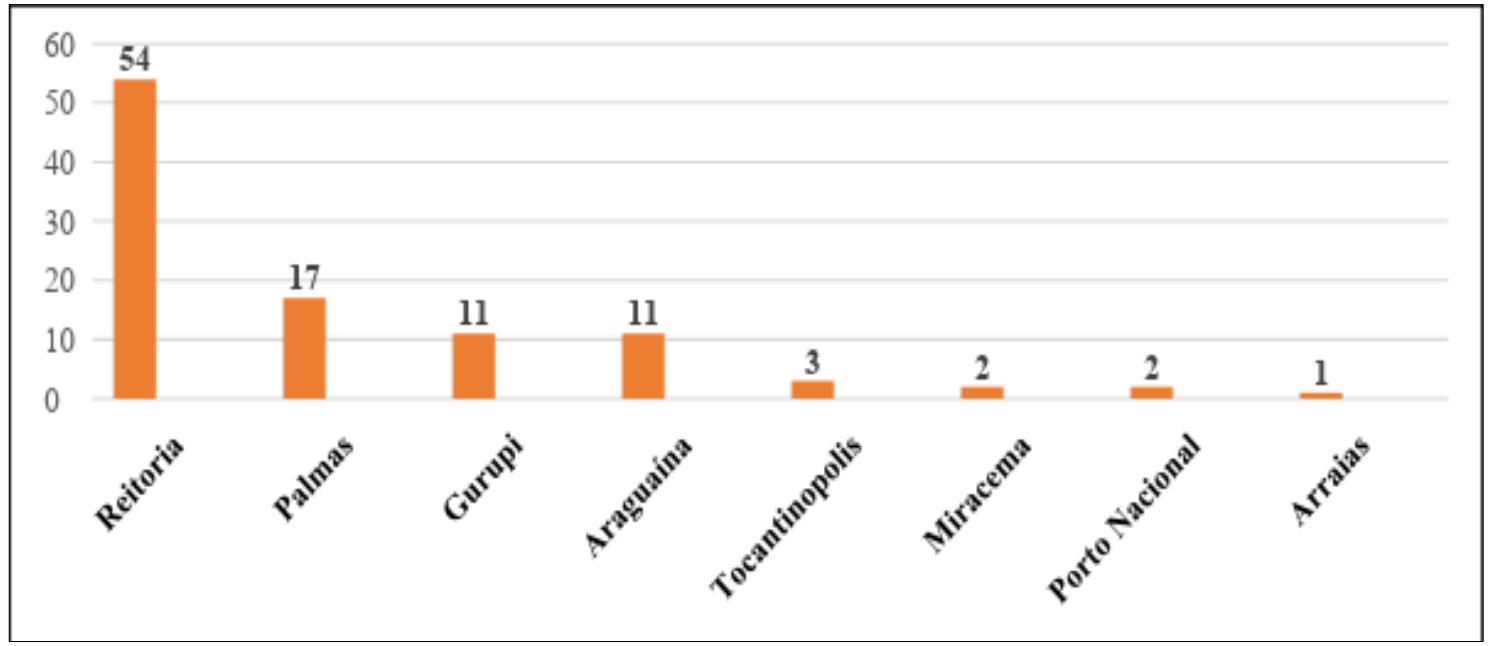

Fonte: Adaptado de UFT (2018).

Observa-se que no geral, a maior parte dos cursos foi ofertado pela Reitoria. Destaca-se, porém, que a maioria das ações de capacitação promovidas pela Reitoria da UFT conta com a participação de servidores lotados nos campi. Foram oferecidos em cada campus, pelo menos um curso de capacitação em diferentes modalidades, contudo, observou-se que a grande maioria, ou mais de $70 \%$ dos cursos foram ofertados na modalidade de capacitação interna, por meio do Servidor Multiplicador (UFT, 2018).

Destarte, infere-se que a UFT apresenta um programa de capacitações articulando uma política de desenvolvimento de seu quadro de pessoal, possivelmente, no intuito de dirimir as lacunas existentes e alcançar os objetivos institucionais, e para isso, aproveita os conhecimentos do seu próprio quadro de pessoal.

Ao analisar quais dos cursos ofertados possuíam relação com a sustentabilidade, destacaram-se 11 cursos de capacitação oferecidos em seis dos sete campi, com exceção do campus de Arraias. Observou-se que alguns cursos foram ofertados em mais de um Câmpus. Destacou-se, ainda, que os cursos estavam relacionados à relevância social, ambiental e econômica da Instituição.

Na Tabela 2, tem-se a distribuição dos cursos identificados com alguma relação com a temática sustentável, conforme a A3P, a IN no 10/2012 e a literatura especializada sobre o tema. 
Tabela 2. Distribuição de cursos ofertados na UFT que possuem relação com a sustentabilidade.

\begin{tabular}{|l|c|}
\hline Curso & Campus \\
\hline $\begin{array}{l}\text { Assessoria da Gestão da Informação e Assentamento } \\
\text { Funcional }\end{array}$ & Reitoria \\
\hline XVI Semana Orçamentária & Reitoria \\
\hline Capacitação para servidores da área Laboratorial & Reitoria \\
\hline Curso de Primeiros Socorros & Araguaína \\
\hline Trabalho em equipe e Gestão de Conflitos no setor Público & Praguaína \\
\hline Curso Organização e Gestão de Arquivos e Documentos \\
\hline Curso Trabalho em Equipe - Flexibilidade, Iniciativa e Líder & Palmas \\
\hline Curso Organização no ambiente de Trabalho & Gurupi \\
\hline Aprender a lidar com a Diversidade Socioeconômica e Cultural & Gurupi \\
\hline Trabalho em equipe e Gestão de Conflitos no setor Público & Porto Nacional \\
\hline Trabalho em equipe e Gestão de Conflitos no setor Público & Miracema \\
\hline
\end{tabular}

Fonte: Adaptado de UFT (2018).

Destacam-se, cursos ofertados na área de qualidade de vida e bem-estar no ambiente de trabalho. Analisa-se, ainda, que há cursos voltados para o bom desempenho orçamentário no Setor Público, e Gestão ótima dos recursos materiais utilizados na Universidade.

De acordo, com IN no 10/2012, além da capacitação dos recursos humanos, visa-se $\mathrm{o}$ atendimento de abordagens voltadas para a qualidade de vida e saúde no ambiente laboral, a gestão adequada de resíduos sólidos e perigosos, a utilização racionalizada de materiais de expediente, bem como, licitações e compras de itens sustentáveis (Brasil, 2012).

Portanto, cabe destaque para os cursos identificados com relação à sustentabilidade, tendo em vista que os temas abordados são direcionados para propostas de melhorias no ambiente de trabalho, visando, mesmo que indiretamente, a atender aos princípios de sustentabilidade. Para Fouto (2002), a universidade deve estabelecer princípios através da implementação de práticas que sirvam de exemplos para a comunidade local e internacional.

Como pontos de análise gerais, averiguou-se que a UFT possui um programa de capacitação de seu quadro de servidores bem planejado e regulamentado, através de Resoluções e mecanismos de planejamento (PFD). Destaca-se, principalmente, que o mapeamento das competências a serem sanadas é baseado no diagnóstico individual de cada servidor.

Como fatores positivos no planejamento e previsão de oferta dos cursos, destacam-se, o mapeamento realizado junto às necessidades individuais e setoriais, porém, ressalta-se o grande número de cursos propostos, aquém da realidade de ofertas. Assim, quando se analisa se as temáticas previstas para oferta se voltam para questões socioambientais, percebe-se que ainda são poucos os cursos previstos no planejamento, e, embora haja a previsão de cursos relacionados, ainda deixam a desejar, se observarmos o que mencionam as diretrizes e políticas governamentais, na A3P e IN no 10/2012.

Com relação à oferta de cursos de capacitação na UFT, ressalta-se, que houve oferta de cursos em todas as unidades. Contudo, a oferta destoa muito do que fora planejado nos PFD. Salientase, que alguns campi possuem dificuldades na oferta de cursos de capacitação por não contar com quadro de pessoal com habilidades para ofertar cursos na modalidade de capacitação interna. Outro fator que pode estar associado à baixa oferta de cursos nesses campi, é a distância que os polos estão 
localizados, e também, as limitações financeiras, o que dificulta a contratação de cursos de capacitação por empresas externas à UFT.

Por fim, ao se analisar os cursos sobre questões socioambientais, observaram-se de positivo que alguns cursos foram ofertados em todos os campi, exceto no campus de Arraias. Entretanto, embora se perceba a sensibilização institucional na oferta de cursos sobre questões tão pertinentes, salienta-se que os cursos ofertados ainda não estão diretamente associados às questões sustentáveis. Neste sentido, cabe à UFT repensar sobre a oferta de cursos mais específicos, visando capacitar seu quadro de pessoal sobre uma temática tão importante $\mathrm{e}$ emergente na atualidade.

\section{Considerações finais}

Este artigo buscou analisar se a Universidade Federal do Tocantins tem se sensibilizado na oferta de capacitação aos seus servidores com temáticas voltadas para a sustentabilidade.

Como principais resultados, destaca-se, que a UFT possui um programa de capacitação elaborado em todos os campus, com previsão e oferta de cursos aos servidores, e baseado nas lacunas informadas pelos próprios servidores. Com relação aos cursos voltados às temáticas socioambientais, apesar de serem previstos no planejamento e ofertados alguns cursos de capacitação relacionados ao tema, entende-se que a Instituição pode elaborar um programa de cursos mais específicos sobre sustentabilidade para a formação de seu quadro de pessoal, considerando a importância do tema.

0 estudo possui algumas limitações, principalmente, no que diz respeito ao acesso ao cronograma dos conteúdos trabalhados nos cursos, para se verificar a proximidade dos mesmos com temáticas socioambientais. Por outro lado, destaca-se como uma abordagem fundamental para a
Administração Pública em todos os seus órgãos, em especial à UFT, por refletir sobre práticas de capacitação e a sustentabilidade, que são fundamentais para o bom desempenho institucional, nos contextos da atualidade.

Como sugestões de melhoria, embora a UFT elabore seus planos com base nas necessidades dos servidores, e promova as ações de capacitação, percebe-se, ainda, um distanciamento entre o que se planeja e o que é ofertado. Com relação à sustentabilidade, compreende-se que apesar de possuir alguns cursos com temáticas sustentáveis, ainda podem ser realizados planos e ações mais específicas sobre a temática. Neste sentido, novos estudos podem ser elaborados para averiguar esse desenvolvimento, ou mesmo uma pesquisa empírica com os sujeitos participantes das ações de capacitação para verificar a efetividade desses programas.

\section{Conflito de interesses} de interesses.

0 autor declara não haver conflito

\section{Referências}

Beuren, I. M. Como elaborar trabalhos monográficos em contabilidade: teoria e prática. 3. ed. São Paulo: Atlas, 2006.

Boyatzis, R. E. The competent manager: A model for effective performance. New York: John Wiley \& Sons, 1982.

Brasil. Lei no 11.091, de 12 de janeiro de 2005. Dispõe sobre a estruturação do Plano de Carreira dos Cargos TécnicoAdministrativos em Educação, no âmbito das Instituições Federais de Ensino vinculadas ao Ministério da Educação, e dá outras providências. Disponível em: <http://www.planalto.gov.br/ccivil_03/_ato2 004-2006/2005/lei/l11091.htm>. Acesso em: 15 jan. 2018.

Brasil. Ministério do Meio Ambiente (MMA). A3P - Agenda Ambiental na Administração Pública. 5. ed. Brasília: MMA, 2009. Disponível em: <http://www.mma.gov.br/ 
estruturas/a3p/_arquivos/cartilha_a3p_36.p df>. Acesso em: 02 jul. 2018.

Brasil. Ministério do Planejamento, Desenvolvimento e Gestão (MPDG). Secretaria de Logística e Tecnologia da Informação (SLTI). Instrução Normativa no 10, de 12 de novembro de 2012. Estabelece regras para elaboração dos Planos de Gestão de Logística Sustentável de que trata o art. 16, do Decreto no 7.746 , de 5 de junho de 2012, e dá outras providências. Disponível em: <https://www.comprasgovernamentais. gov.br/index.php/legislacao/instrucoesnormativas/394-instrucao-normativa-n-10de-12-de-novembro-de-2012>. Acesso em: 22 jun. 2017.

CMMAD - Comissão Mundial sobre Meio Ambiente e Desenvolvimento. Nosso futuro comum. Rio de Janeiro: Fundação Getúlio Vargas, 1988.

Cole, L. Assessing sustainability on Canadian university campuses: Development of a campus sustainability assessment framework. Victoria: Royal Roads University, 2003. (Dissertation).

Dutra, J.S. Competências: conceitos e instrumentos para a gestão de pessoas na empresa moderna. São Paulo: Atlas, 2004.

Fleury, M. T. L.; Fleury, A. Construindo o conceito de competência. Revista de Administração Contemporânea - RAC, v. 5, ed. esp., p. 183-196, 2001. Disponível em: <http://www.scielo.br/pdf/rac/v5nspe/v5n spea10.pdf>. Acesso em: 20 nov. 2017.

Fouto, A.R.F. 0 papel das universidades rumo ao desenvolvimento sustentável: das relações internacionais às práticas locais. Lisboa: Universidade Nova, 2002. (Dissertação).

Gil, A. C. Métodos e técnicas de pesquisa social. 6. ed. São Paulo: Atlas, 2008.

Le Boterf, G. Desenvolvendo a competência dos profissionais. 3. ed. Porto Alegre: Artmed, 2003.

McClelland, D.C. Testing for competence rather than for "intelligence". American Psychologist, v. 28, p. 1-14, 1973.

Prodanov, C. C.; Freitas, E. C. Metodologia do trabalho científico: métodos e técnicas da pesquisa e do trabalho acadêmico. 2. ed. Novo Hamburgo: Feevale, 2013.
Quadros, D. S. Subsídios para o sistema de gestão ambiental da Universidade Regional de Blumenau. Blumenau: Universidade Regional de Blumenau, 1999. (Dissertação).

Sachs, I. Desenvolvimento: includente, sustentável, sustentado. Rio de Janeiro: Garamond, 2008.

Sachs, I. Caminhos para o desenvolvimento sustentável. Rio de Janeiro: Garamond, 2009.

Schikmann, R. Gestão estratégica de pessoas: bases para a concepção do Curso de Especialização em Gestão de Pessoas no Serviço Público. In: Camões, M. R. S.; Pantoja, M. J.; Bergue, S. T. (Org.). Gestão de pessoas: bases teóricas e experiências no setor público. Brasília: ENAP, 2010.

Silva, G. S.; Silva, A. C. Gestão de pessoas no serviço público: os programas de capacitação de servidores técnico-administrativos na Universidade Federal do Tocantins - Campus de Araguaína, em 2016. Revista Estudo \& Debate, v. 25, n. 2, p. 41-65, 2018. https://doi.org/10.22410/issn.1983-036X. v25i2a2018.1560

Souza, V. D.; Uhlmann, V. O.; Casagrande, M. D. H. Sustentabilidade ambiental em instituição de ensino: uma investigação da aderência à Agenda Ambiental de Administração Pública - A3P. Anais do XIII Encontro Nacional Sobre Gestão Empresarial e Meio Ambiente, São Paulo, 2011.

UFT - Universidade Federal do Tocantins. Resolução do Conselho Universitário no 20/2015. Disponível em: <http://docs.uft. edu.br/share/s/Y3uvFGraQRa7nQAsLlfl2w>. Acesso em: 21 fev. 2017.

UFT - Universidade Federal do Tocantins. Plano de Desenvolvimento Institucional 2016-2020. 2016. Disponível em: <http://docs.uft.edu.br/share/s/Bu0fAqZjT6 6B-rTgwt53LQ>. Acesso em: 21 fev. 2018.

UFT - Universidade Federal do Tocantins. Plano de formação e desenvolvimento dos servidores técnico-administrativos da Universidade Federal do Tocantins Biênio 2017-2018. 2017. Disponível em: <http://ww2.uft.edu.br/gestao/proreitorias/progedep>. Acesso em: 05 maio 2018. 
UFT - Universidade Federal do Tocantins. Sistema de Informações para o Ensino (SIE). Relatório dos cursos de capacitação/ treinamentos. Araguaína. 2018. Disponível em: <http://termarag.uft.edu.br>. Acesso em: 19 abr. 2018.

Zarifian, P. Objetivo competência: por uma nova lógica. São Paulo: Atlas, 2001. seja devidamente citada. 